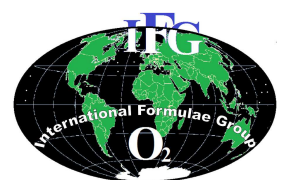

\title{
Détermination du débit de filtration glomérulaire (DFG) au cours du diabète : Cockroft et Gault, MDRD ou CKD-EPI ?
}

\author{
El Hadji Malick NDOUR ${ }^{1}$, Fatou GUEYE TALL ${ }^{1}$, Anna SARR ${ }^{2}$, \\ Papa Madièye GUEYE ${ }^{1}$, Hélène Ange Thérèse SAGNA ${ }^{1}$, \\ Djibril Mamadou COULIBALY ${ }^{1}$, Rokhaya NDIAYE DIALLO ${ }^{1}$, \\ Papa Amadou DIOP ${ }^{1}$, Philomène LOPEZ SALL ${ }^{1}$ et Aynina CISSE ${ }^{1 *}$ \\ ${ }^{1}$ Laboratoire de Biochimie Pharmaceutique, Faculté de Médecine, de Pharmacie et d'Odontologie, \\ Université Cheikh Anta Diop de Dakar, Sénégal. \\ ${ }^{2}$ Clinique de Médecine Interne II, Faculté de Médecine, de Pharmacie et d'Odontologie, \\ Université Cheikh Anta Diop de Dakar, Sénégal. \\ *Auteur correspondant ; E-mail: aycisse@yahoo.fr
}

\section{RESUME}

Plusieurs paramètres peuvent être étudiés pour évaluer le rein. Parmi ceux-ci, le débit de filtration glomérulaire (DFG) a été déterminé avec les formules de Cockroft et Gault (CG), du Modification of Diet in Renal Disease (MDRD) et du Chronic Kidney Disease EPIdemiology Collaboration (CKD-EPI) et la formule la mieux adaptée pour le diabétique a été recherchée. Chez 59 diabétiques de type 1 (DT1) et 70 diabétiques de type 2 (DT2), le DFG a été déterminé avec les formules de CG, du MDRD et du CKD-EPI. Avec l'analyse statistique, les seuils de significativité ont été fixés pour $\mathrm{p}<0,05 ; \mathrm{T}_{0} \alpha>1,96$ et $\mathrm{Z}_{0} \alpha>1,96$. Le MDRD est superposable au CKD-EPI chez les DT1 et DT2. Chez les DT1, le DFG moyen et la corrélation entre $1 /$ créatininémie et DFG ne varient pas si CG ou CKD-EPI ; cependant, les sujets à DFG réduit $(<90$ $\left.\mathrm{ml} / \mathrm{min} / 1,73 \mathrm{~m}^{2}\right)$ sont plus nombreux avec CG plutôt qu'avec CKD-EPI $\left(66,10 \%\right.$ vs $\left.47,46 \% ; \mathrm{T}_{0} \alpha=2,05\right)$. Chez les DT2, le DFG moyen et la proportion de sujets à DFG réduit sont indépendants de la formule utilisée, mais la corrélation entre 1 /créatininémie et DFG est plus forte si CKD-EPI que CG $\left(0,961\right.$ vs 0,$\left.632 ; Z_{0} \alpha=7,02\right)$. Ainsi, la formule la mieux adaptée pour la détermination du DFG serait CG chez les DT1 et CKD-EPI chez les DT2, sachant que CKD-EPI est équivalent à MDRD quel que soit le type de diabète.

(C) 2015 International Formulae Group. All rights reserved.

Mots clés : Cockroft et Gault - MDRD - CKD-EPI - débit de filtration glomérulaire (DFG) - diabète.

\section{INTRODUCTION}

Le diabète est une hyperglycémie chronique résultant d'un déficit quantitatif (diabète de type 1) et/ou qualitatif en insuline (diabète de type 2). Il peut être à l'origine de complications chroniques à titre de macroangiopathies et de microangiopathies.
Parmi les complications microangiopathiques, on note la néphropathie diabétique (Battisti et al., 2003).

La néphropathie diabétique affecte 25 à $40 \%$ des patients diabétiques et le diabète est la cause principale d'insuffisance rénale terminale dans les pays développés. La 
proportion de diabétiques à insuffisance rénale terminale augmente de façon dramatique avec l'augmentation de l'espérance de vie des diabétiques de type 2 . Les taux de survie sont faibles chez ces patients à cause du risque cardio-vasculaire élevé (Rigalleau et al., 2005).

Selon les statistiques des Etats-Unis et d'autres pays, le nombre de patients atteints de maladie rénale a doublé entre 2003 et 2010 (Tomáš et al., 2014). Les patients atteints d'insuffisance rénale chronique (IRC) représentent 7-10\% de la population mondiale (Xioguang et al., 2014). L'IRC est devenue un problème de santé publique. La maladie rénale chronique reste asymptomatique jusqu'au stade tardif où l'intervention ne peut plus arrêter la progression de la maladie. Par conséquent, il est urgent de diagnostiquer précocement cette maladie (Yasmin et Hasniza, 2015).

Les paramètres pour le diagnostic et la surveillance de la maladie rénale chronique chez les patients souffrant de diabète sont la microalbuminurie et le débit de filtration glomérulaire (DFG) (Rigalleau et al., 2011 ).

La microalbuminurie précéderait la diminution du DFG chez les sujets diabétiques. Toutefois, certaines études montrent que la baisse du DFG accompagne souvent la microalbuminurie (Halimi, 2012) ou peut survenir chez un diabétique normoalbuminurique (Jerums et al., 2012).

Le DFG est défini comme la quantité de plasma qui traverse le glomérule rénal par unité de temps. Il est considéré comme le meilleur indice global de la fonction rénale (Tomáš et al., 2014). Pour déterminer le DFG, la clairance de marqueurs exogènes (inuline, iohexol, acide éthylène diamine tétraacétique) ou de marqueurs endogènes (urée, créatine) peut être utilisée (Stevens et al., 2006; Hermida-Cadahia et al., 2012). La détermination du DFG par la mesure de la clairance des marqueurs exogènes est la méthode de référence mais elle est onéreuse, elle prend beaucoup de temps et elle est de réalisation technique difficile. Dès lors, elle ne peut être envisagée en pratique quotidienne (Levey et al., 2009).

La substance endogène la plus utilisée pour déterminer le DFG en routine est la créatinine. La créatininémie $(\mathrm{Cr})$ est un bon reflet de la filtration glomérulaire. Cependant, elle n'est pas un marqueur idéal du DFG. La Cr dépend aussi de la production musculaire de créatinine et de sa sécrétion tubulaire (Rigalleau et al., 2011). Ainsi la clairance de la créatinine endogène $(\mathrm{Clcr})$ est considérée comme un meilleur marqueur de la fonction rénale, comparée à la créatininémie (Tomáš et al., 2014). La détermination de la Clcr est de réalisation aisée mais elle est liée à des difficultés notamment celles relatives à la collecte des urines de 24 heures, à l'origine d'erreurs grossières (Earley et al., 2012).

Aussi, plusieurs formules ont-elles été élaborées pour estimer le DFG chez les adultes. Il s'agit notamment de la formule de Cockroft et Gault (CG) (1976), de la formule du Modification of Diet in Renal Disease (MDRD) simplifiée (Levey et al., 2009) et de la formule du Chronic Kidney Disease EPIdemiology (CKD-EPI) (Levey et al., 2009). Ces formules intègrent des données anthropométriques avec des incertitudes particulières pour les patients diabétiques liées au large éventail de DFG, à l'index de masse corporelle (IMC) et à l'effet propre de la glycémie (Rigalleau et al., 2011).

Ainsi, l'objectif de ce travail est-il de rechercher parmi ces trois formules la mieux adaptée pour déterminer le DFG chez les diabétiques de type 1 (DT1) d'une part et chez les diabétiques de type 2 (DT2) d'autre part.

\section{MATERIEL ET METHODES}

Il s'agissait d'une étude analytique d'observation prospective. L'étude a porté sur 58 sujets non diabétiques, sur 59 DT1 et 70 DT2. 
Le recrutement des diabétiques a été effectué, au tout venant, au centre anti-diabète Marc Sankalé sis à l'hôpital Abass Ndao de Dakar (Sénégal). Pour les témoins, il a eu lieu au laboratoire de biologie médicale de l'Hôpital d'Enfants Albert Royer (HEAR) de Dakar (Sénégal). Le type de diabète a été précisé à l'aide du dossier médical. Ce sont tous des sénégalais de race noire de type subsaharien. Ils ont participé à l'étude après un consentement éclairé.

La glycémie a été déterminée avec la méthode à la glucose oxydase/peroxydase à l'aide du semi-automate Biosystems BTS 350 (Biosystems Reagents \& Instruments, Costa Brava, Barcelona, Spain).

La créatininémie a été déterminée avec la méthode de Jaffé, en cinétique, à l'aide du semi-automate Hospitex master (Hospitex Diagnostics, Firenza, Italy) en utilisant le kit créatinine/picrate alcalin ((Biosystems Reagents \& Instruments, Barcelona, Spain).

L'indice de masse corporelle (IMC) et l'indice de masse grasse (IMG) ont été respectivement calculés à l'aide de la formule de Quételet (Batsis et al., 2014) et à l'aide de la formule de Deurenberg et al. (1991).

Le DFG a été déterminé avec les formules de Cockroft et Gault (1976), du MDRD simplifiée (Levey et al., 2009) et du CKD-EPI (Levey et al., 2009).

\section{Analyse statistique}

L'analyse statistique a été réalisée à l'aide de la version académique 2007 du logiciel Microsoft Excel (Microsoft, Redmond, USA).

Les moyennes ont été comparées avec le test $\mathrm{t}$ de Student, les coefficients de corrélation de Pearson avec le test $\mathrm{Z}$ après transformation de Fisher.

Les différences de moyenne, de proportions et de coefficients de corrélation ont été respectivement considérées comme statistiquement significatives pour des valeurs de $\mathrm{p}<0,05 ;$ de $\mathrm{T}_{0} \alpha>1,96$ et de $Z_{0 \alpha}>1,96$.

\section{RESULTATS}

Les sujets DT1 et les témoins ont statistiquement le même âge. Les moyennes de leurs IMC sont superposables (Tableau 1).

Comparés aux DT2, les DT1 sont moins âgés. La moyenne de l'IMC des DT1 est basse, comparée à celle des DT2. Par contre, la durée moyenne du diabète est plus longue chez les DT1 par rapport aux DT2 (Tableau 2).

Les moyennes de la glycémie et de la créatininémie sont superposables entre témoins et DT1. De même, il n'y a pas de différence statistiquement significative entre témoins et DT1 quelle que soit la formule utilisée pour l'estimation du DFG (Tableau 3).

La moyenne de la glycémie est superposable entre les DT1 et les DT2. Les moyennes de la créatininémie des DT2 et des DT1 ne montrent pas de différence statistiquement significative. Quelle que soit la formule utilisée, les moyennes des DFG des DT2 sont basses comparées à celles des DT1 (Tableau 4).

Chez les sujets diabétiques de type 1 , il est apparu que :

La formule du CKD-EPI et celle du MDRD modifié donnent des moyennes de DFG superposables (respectivement $91,11 \pm 25,91$ et $89,96 \pm 26,21 \mathrm{ml} / \mathrm{min} / 1,73 \mathrm{~m}^{2}$; $\mathrm{p}=0,81$ ) (Tableau 5).

La formule du CKD-EPI donne exactement la même proportion de sujets à DFG réduit (i.e. $<90 \mathrm{ml} / \mathrm{min} / 1,73 \mathrm{~m}^{2}$ ) que la formule du MDRD modifiée (47,46\% versus $47,46 \% ; \mathrm{T}_{0} \alpha=0$ ) (Tableau 6).

Lorsque les DFG sont inférieurs à 90 $\mathrm{ml} / \mathrm{min} / 1,73 \mathrm{~m}^{2}$, la corrélation entre le $1 / \mathrm{Cr}$ et le DFG n'est pas significative si le DFG est calculé avec la formule du CKD-EPI au lieu de la formule du MDRD simplifiée $(0,865$ versus 0,$858 ; Z_{0} \alpha=0,14$ ) (Tableau 7; Tableau 8).

Par ailleurs, chez les sujets diabétiques de type 1 , il est aussi mis en évidence que : 
- DFG-CG et DFG-CKD-EPI sont superposables $\left(82,61 \pm 26,57 \mathrm{ml} / \mathrm{min} / 1,73 \mathrm{~m}^{2}\right.$ versus $91,11 \pm 25,91 \mathrm{ml} / \mathrm{min} / 1,73 \mathrm{~m}^{2} ; \mathrm{p}=$ $0,81)$.

- $\quad$ La proportion de sujets à DFG $<90$ $\mathrm{ml} / \mathrm{min} / 1,73 \mathrm{~m}^{2}$ est statistiquement plus importante quand le DFG est déterminé à l'aide de la formule de Cockroft et Gault plutôt qu'à l'aide de la formule du CKD-EPI $\left(66,10 \%\right.$ versus $\left.47,46 \% ; \quad \mathrm{T}_{0} \alpha=2,05\right)$ (Tableau 9).

Lorsque les DFG calculés avec la formule du CKD-EPI sont inférieurs à 90 $\mathrm{ml} / \mathrm{min} / 1,73 \mathrm{~m}^{2}$, la corrélation entre $1 / \mathrm{Cr}$ et DFG est de $r=0,865$. Cette corrélation ne présente pas de différence statistiquement significative avec celle obtenue avec la formule de $C G(r=0,793)\left(Z_{0} \alpha=1,23\right)$ (Tableau 10).

En ce qui concerne les sujets diabétiques de type 2 :

- $\quad$ La formule du CKD-EPI et celle du MDRD modifié donnent des valeurs moyennes du DFG superposables (76,23 $\pm 19,06$ versus $78,61 \pm 19,04 \mathrm{ml} / \mathrm{min} / 1,73$ $\left.\mathrm{m}^{2} ; \mathrm{p}=0,46\right)$ (Tableau 11). Ces formules conduisent aussi aux mêmes proportions de sujets à $\mathrm{DFG}<90 \mathrm{ml} / \mathrm{min} / 1,73 \mathrm{~m}^{2}(80 \%$ versus 80\%; $\mathrm{T}_{0} \alpha=00$ ) (Tableau 12).
Lorsque les DFG déterminés avec la formule du CKD-EPI ou celle du MDRD modifiée, il n'y a pas de différence statistiquement significative pour la corrélation entre $1 / \mathrm{Cr}$ et DFG $(0,787$ versus 0,$817 ; \mathrm{Z}_{0} \alpha=0,49$ ) (Tableaux 13; 14).

Par ailleurs, la formule de CG donne une valeur moyenne du DFG statistiquement superposable à celle obtenue avec la formule du CKD-EPI $(71,87+/-24,23$ versus 76,23+/$\left.19,06 \mathrm{ml} / \mathrm{min} / 1,73 \mathrm{~m}^{2} ; \mathrm{p}=0,24\right)$. De même, la proportion de sujets à $\mathrm{DFG}<90$ $\mathrm{ml} / \mathrm{min} / 1,73 \mathrm{~m}^{2}$ obtenue avec la formule $\mathrm{du}$ CKD-EPI est superposable $\quad(80 \%$ versus $\left.78,57 \% ; \mathrm{T}_{0} \alpha=0,21\right)$ à celle obtenue avec la formule de Cockroft et Gault (Tableau 15) et il est observé une meilleure corrélation entre $1 / \mathrm{Cr}$ et DFG pour CKD-EPI par rapport à CG $\left(0,787\right.$ versus 0,$\left.545 ; \mathrm{Z}_{0} \alpha=2,61\right)$ (Tableau 16).

Si les DFG sont compris entre 90 et 60 $\mathrm{ml} / \mathrm{min} / 1,73 \mathrm{~m}^{2}$, la corrélation avec $1 / \mathrm{Cr}$ devient presque inexistante $(r=0,157)$ en utilisant $\mathrm{CG}$ par rapport à celle obtenue avec CKD-EPI. Par contre, lorsque les DFG sont déterminés avec la formule du CKD-EPI $\left(0,157\right.$ versus 0,$\left.526 ; \mathrm{Z}_{0} \alpha=2,46\right)$ (Tableau 16).

Tableau 1: Comparaison des variables anthropométriques entre les diabétiques de type 1 et les témoins.

\begin{tabular}{lccc}
\hline & DT1 & Témoins & p-value $<\mathbf{0 , 0 5}$ \\
\hline Age $($ ans) & $35,39+/-11,28$ & $35,6+/-12,91$ & 0,92 \\
IMC $\left(\mathrm{kg} / \mathrm{m}^{2}\right)$ & $22,61+/-3,92$ & $23,39+/-4,93$ & 0,34 \\
Tour de taille $(\mathrm{cm})$ & $81,61+/-10,51$ & $75+/-14,17$ & 0,89 \\
Tour de hanche $(\mathrm{cm})$ & $97,32+/-10,51$ & $98,08+/-12,04$ & 0,70 \\
sexe ratio $(\mathrm{H} / \mathrm{F})$ & 0,64 & 0,81 & \\
\hline
\end{tabular}


Tableau 2: Comparaison des variables anthropométriques entre diabétiques de type 1 et diabétiques de type 2 .

\begin{tabular}{lccc}
\hline & DT1 & DT2 & p-value<0,05 \\
\hline Age (ans) & $35,39+/-11,28$ & $58,86+/-9,93$ & $5,03.10^{-24}$ \\
Poids (Kg) & $64,68 \pm 10,71$ & $71,8 \pm 13,23$ & $1,2.10^{-3}$ \\
IMC $\left(\mathrm{kg} / \mathrm{m}^{2}\right)$ & $22,61+/-3,92$ & $25,77+/-4,84$ & 0,000098 \\
IMG $(\%)$ & $25,66 \pm 9,35 \%$ & $36,90 \pm 7,27 \%$ & $3,87.10^{-12}$ \\
Tour de taille (cm) & $81,61+/-10,51$ & $89,835+/-11,98$ & 0,000095 \\
Tour de hanche (cm) & $97,32+/-10,51$ & $104,24+/-11,69$ & 0,00063 \\
Ancienneté (ans) & $11,46+/-6,51$ & $8,01+/-5,38$ & 0,0012 \\
sexe ratio (H/F) & 0,64 & 0,25 & \\
\hline
\end{tabular}

Tableau 3: Comparaison des variables biochimiques entre diabétiques de type 1 et témoins.

\begin{tabular}{lccc}
\hline & DT1 $(\mathbf{n = 5 9})$ & Témoin $(\mathbf{n = 5 8})$ & p-value $<\mathbf{0 , 0 5}$ \\
\hline Glycémie $(\mathrm{g} / \mathrm{l})$ & $1,99+/-0,94$ & $0,85+/-0,18$ & $2,31.10^{-15}$ \\
Créatininémie $(\mu \mathrm{mol} / \mathrm{l})$ & $114,47+/-152,79$ & $95,34+/-18,94$ & 0,34 \\
CG $\left(\mathrm{ml} / \mathrm{min} / 1,73 \mathrm{~m}^{2}\right)$ & $82,61+/-26,57$ & $84,88+/-20,09$ & 0,59 \\
MDRD $\left(\mathrm{ml} / \mathrm{min} / 1,73 \mathrm{~m}^{2}\right)$ & $89,96+/-26,21$ & $89,67+/-17,53$ & 0,97 \\
CKD-EPI $\left(\mathrm{ml} / \mathrm{min} / 1,73 \mathrm{~m}^{2}\right)$ & $91,11+/-25,91$ & $91,18+/-18,81$ & 0,96 \\
\hline
\end{tabular}

Tableau 4: Comparaison des variables biochimiques entre diabétiques de type 1 et diabétiques de type 2 .

\begin{tabular}{lccc}
\hline & DT1 $(\mathbf{n = 5 9})$ & DT2 $(\mathbf{n}=\mathbf{7 0})$ & p-value $<\mathbf{0 , 0 5}$ \\
\hline Glycémie $(\mathrm{g} / \mathrm{l})$ & $1,99+/-0,94$ & $1,70+/-0,79$ & $6,5.10^{-2}$ \\
Créatininémie $(\mu \mathrm{mol} / \mathrm{l})$ & $114,47+/-152,79$ & $91,83+/-22,05$ & $2,6.10^{-1}$ \\
DFG-CG $\left(\mathrm{ml} / \mathrm{min} / 1,73 \mathrm{~m}^{2}\right)$ & $82,61+/-26,57$ & $71,87+/-24,23$ & $1,8.10^{-2}$ \\
DFG-MDRD $\left(\mathrm{ml} / \mathrm{min} / 1,73 \mathrm{~m}^{2}\right)$ & $89,96+/-26,21$ & $78,61+/-19,04$ & $5,2.10^{-3}$ \\
DFG-CKD-EPI $\left(\mathrm{ml} / \mathrm{min} / 1,73 \mathrm{~m}^{2}\right)$ & $91,11+/-25,91$ & $76,23+/-19,06$ & $2,7.10^{-4}$ \\
\hline
\end{tabular}

Tableau 5 : Débit de filtration glomérulaire (DFG) chez les diabétiques de type 1.

\begin{tabular}{lccc}
\hline & \multicolumn{3}{c}{ DT1 (59) } \\
\hline DFG] $0 ; 160 \mathrm{ml} / \mathrm{min} / 1,73 \mathrm{~m}^{2}[$ & $82,61+/-26,57$ & $89,96+/-26,21$ & $91,11+/-25,91$ \\
DFG $<90 \mathrm{ml} / \mathrm{min} / 1,73 \mathrm{~m}^{2}$ & $68,25+/-16,95$ & $68,89+/-18,35$ & $69,63+/-19,25$ \\
DFG $<60 \mathrm{ml} / \mathrm{min} / 1,73 \mathrm{~m}^{2}$ & $46,40+/-16,14$ & $41,57+/-20,93$ & $41,57+/-20,93$ \\
DFG $\left[60 ; 90 \mathrm{ml} / \mathrm{min} / 1,73 \mathrm{~m}^{2}[\right.$ & $75,79+/-8,77$ & $76,17+/-8,17$ & $77,28+/-9,27$ \\
DFG $\geq 90 \mathrm{ml} / \mathrm{min} / 1,73 \mathrm{~m}^{2}$ & $110,61+/-18,23$ & $108,99+/-15,38$ & $110,52+/-12,00$ \\
\hline
\end{tabular}


Tableau 6 : Comparaison des fréquences (\%) des valeurs du DFG chez les sujets diabétiques de type 1avec les formules du MDRD et du CKD-EPI.

\begin{tabular}{lccc}
\hline & \multicolumn{3}{c}{ DT1 (n=59) } \\
\hline & MDRD & CKD-EPI & $\mathbf{T}_{\mathbf{0}} \boldsymbol{\alpha}>\mathbf{1 , 9 6}$ \\
\hline $\mathrm{DFG} \geq 90 \mathrm{ml} / \mathrm{min} / 1,73 \mathrm{~m}^{2}$ & $52,54 \%$ & $52,54 \%$ & 0 \\
$\mathrm{DFG}<90 \mathrm{ml} / \mathrm{min} / 1,73 \mathrm{~m}^{2}$ & $47,46 \%$ & $47,46 \%$ & 0 \\
DFG $\left[60 ; 90 \mathrm{ml} / \mathrm{min} / 1,73 \mathrm{~m}^{2}[\right.$ & $37,29 \%$ & $37,29 \%$ & 0 \\
\hline
\end{tabular}

Tableau 7 : Inverse de la créatininémie (1/Cr) chez les diabétiques de type 1.

\begin{tabular}{lccc}
\hline & \multicolumn{3}{c}{ DT1 (59) } \\
\hline & CG & MDRD & CKD-EPI \\
\hline DFG] $0 ; 160 \mathrm{ml} / \mathrm{min} / 1,73 \mathrm{~m}^{2}[$ & $0,011+/-0,011$ & $0,011+/-0,011$ & $0,011+/-0,011$ \\
DFG $<90 \mathrm{ml} / \mathrm{min} / 1,73 \mathrm{~m}^{2}$ & $0,010+/-0,003$ & $0,009+/-0,002$ & $0,009+/-0,002$ \\
DFG $<60 \mathrm{ml} / \mathrm{min} / 1,73 \mathrm{~m}^{2}$ & $0,007+/-0,002$ & $0,006+/-0,003$ & $0,006+/-0,003$ \\
DFG $\left[60 ; 90 \mathrm{ml} / \mathrm{min} / 1,73 \mathrm{~m}^{2}[\right.$ & $0,011+/-0,002$ & $0,010+/-0,0015$ & $0,010+/-0,0015$ \\
DFG $\geq 90 \mathrm{ml} / \mathrm{min} / 1,73 \mathrm{~m}^{2}$ & $0,013+/-0,002$ & $0,013+/-0,002$ & $0,013+/-0,002$ \\
\hline
\end{tabular}

Tableau 8 : Comparaison des coefficients de corrélation $\mathrm{r}$ entre $1 / \mathrm{Cr}(1 / \mu \mathrm{mol})$ et $\mathrm{DFG}$ $\left(\mathrm{ml} / \mathrm{min} / 1,73 \mathrm{~m}^{2}\right)$ obtenu avec le MDRD et le CKD-EPI chez les diabétiques de type 1.

\begin{tabular}{lccc}
\hline KD- & \multicolumn{3}{c}{ DT1 (n=59) } \\
\cline { 2 - 4 } & $\mathrm{r}($ MDRD $; 1 / \mathrm{Cr})$ & $\mathrm{r}(\mathrm{CKD}-\mathrm{EPI} ; 1 / \mathrm{Cr})$ & $\mathrm{Z}_{0} \alpha>1,96$ \\
\hline DFG] $0 ; 160 \mathrm{ml} / \mathrm{min} / 1,73 \mathrm{~m}^{2}[$ & 0,839 & 0,848 & 0,16 \\
DFG $<90 \mathrm{ml} / \mathrm{min} / 1,73 \mathrm{~m}^{2}$ & 0,858 & 0,865 & 0,14 \\
DFG $<60 \mathrm{ml} / \mathrm{min} / 1,73 \mathrm{~m}^{2}$ & 0,937 & 0,939 & 0,09 \\
DFG $\left[60 ; 90 \mathrm{ml} / \mathrm{min} / 1,73 \mathrm{~m}^{2}[\right.$ & 0,481 & 0,526 & 0,32 \\
DFG $\geq 90 \mathrm{ml} / \mathrm{min} / 1,73 \mathrm{~m}^{2}$ & 0,459 & 0,44 & 0,12 \\
\hline
\end{tabular}

Tableau 9: Comparaison des fréquences (\%) des valeurs du DFG chez les sujets diabétiques de type 1avec les formules de CG et du CKD-EPI.

\begin{tabular}{lccc}
\hline \multicolumn{4}{c}{ DT1 (n=59) } \\
\hline & CG & CKD-EPI & $\mathbf{T}_{\mathbf{0}} \boldsymbol{\alpha}>\mathbf{1 , 9 6}$ \\
\hline DFG $\geq 90 \mathrm{ml} / \mathrm{min} / 1,73 \mathrm{~m}^{2}$ & $33,90 \%$ & $52,54 \%$ & 2,05 \\
DFG $<90 \mathrm{ml} / \mathrm{min} / 1,73 \mathrm{~m}^{2}$ & $66,10 \%$ & $47,46 \%$ & 2,05 \\
DFG $\left[60 ; 90 \mathrm{ml} / \mathrm{min} / 1,73 \mathrm{~m}^{2}[\right.$ & $49,15 \%$ & $37,29 \%$ & 1,3 \\
DFG $<60 \mathrm{ml} / \mathrm{min} / 1,73 \mathrm{~m}^{2}$ & $16,95 \%$ & $10,17 \%$ & 1,08 \\
\hline
\end{tabular}


Tableau 10 : Comparaison des coefficients de corrélation entre $1 /$ créatininémie $(1 / \mu \mathrm{mol})$ et DFG (ml/min/1,73 $\left.\mathrm{m}^{2}\right)$ obtenu avec CG et CKD-EPI chez les diabétiques de type 1.

\begin{tabular}{lccc}
\hline & \multicolumn{3}{c}{ DT1 (n=59) } \\
\hline & $\mathrm{r}(\mathrm{CG} ; 1 / \mathrm{Cr})$ & $\mathrm{r}(\mathrm{CKD}-\mathrm{EPI} ; 1 / \mathrm{Cr})$ & $\mathrm{Z}_{0} \alpha>1,96$ \\
\hline DFG] $0 ; 160 \mathrm{ml} / \mathrm{min} / 1,73 \mathrm{~m}^{2}[$ & 0,776 & 0,848 & 1,13 \\
DFG $<90 \mathrm{ml} / \mathrm{min} / 1,73 \mathrm{~m}^{2}$ & 0,793 & 0,865 & 1,23 \\
DFG $<60 \mathrm{ml} / \mathrm{min} / 1,73 \mathrm{~m}^{2}$ & 0,897 & 0,939 & 1,44 \\
DFG $\left[60 ; 90 \mathrm{ml} / \mathrm{min} / 1,73 \mathrm{~m}^{2}[\right.$ & 0,528 & 0,526 & 0,01 \\
DFG $\geq 90 \mathrm{ml} / \mathrm{min} / 1,73 \mathrm{~m}^{2}$ & 0,505 & 0,44 & 0,44 \\
\hline
\end{tabular}

Tableau 11 : Débit de filtration glomérulaire chez les diabétiques de type 2.

\begin{tabular}{lccc}
\hline & \multicolumn{3}{c}{ DT2 (70) } \\
\hline & CG & MDRD & CKD-EPI \\
\hline DFG] $0 ; 160 \mathrm{ml} / \mathrm{min} / 1,73 \mathrm{~m}^{2}[$ & $71,87+/-24,23$ & $78,61+/-19,04$ & $76,23+/-19,06$ \\
DFG $<90 \mathrm{ml} / \mathrm{min} / 1,73 \mathrm{~m}^{2}$ & $61,89+/-14,02$ & $71,36+/-11,69$ & $68,82+/-12,26$ \\
DFG $<60 \mathrm{ml} / \mathrm{min} / 1,73 \mathrm{~m}^{2}$ & $49,27+/-7,83$ & $49,57+/-9,36$ & $50,01+/-9,99$ \\
DFG $\left[60 ; 90 \mathrm{ml} / \mathrm{min} / 1,73 \mathrm{~m}^{2}[\right.$ & $72,40+/-7,99$ & $74,47+/-8,16$ & $72,92+/-8,25$ \\
DFG $\geq 90 \mathrm{ml} / \mathrm{min} / 1,73 \mathrm{~m}^{2}$ & $108,49+/-17,49$ & $107,62+/-14,56$ & $105,87+/-10,49$ \\
\hline
\end{tabular}

Tableau 12: Comparaison des fréquences (\%) des valeurs du DFG chez les sujets diabétiques de type 2 avec les formules du MDRD et du CKD-EPI.

\begin{tabular}{lccc}
\hline & \multicolumn{3}{c}{ DT2 (n= 70) } \\
\hline & MDRD & CKD-EPI & T $_{\mathbf{0}} \boldsymbol{\alpha}>\mathbf{1 , 9 6}$ \\
\hline DFG $\geq 90 \mathrm{ml} / \mathrm{min} / 1,73 \mathrm{~m}^{2}$ & $20 \%$ & $20 \%$ & 0 \\
DFG $<90 \mathrm{ml} / \mathrm{min} / 1,73 \mathrm{~m}^{2}$ & $80 \%$ & $80 \%$ & 0 \\
DFG $\left[60 ; 90 \mathrm{ml} / \mathrm{min} / 1,73 \mathrm{~m}^{2}[\right.$ & $70 \%$ & $65,71 \%$ & 0,54 \\
DFG $<60 \mathrm{ml} / \mathrm{min} / 1,73 \mathrm{~m}^{2}$ & $10 \%$ & $14,29 \%$ & 0,78 \\
\hline
\end{tabular}

Tableau 13: Inverse de la créatininémie chez les diabétiques de type 2.

\begin{tabular}{lccc}
\hline & \multicolumn{3}{c}{ DT2 (70) } \\
\hline & CG & MDRD & CKD-EPI \\
\hline DFG] $0 ; 160 \mathrm{ml} / \mathrm{min} / 1,73 \mathrm{~m}^{2}[$ & $0,01+/-0,002$ & $0,01+/-0,002$ & $0,01+/-0,002$ \\
DFG $<90 \mathrm{ml} / \mathrm{min} / 1,73 \mathrm{~m}^{2}$ & $0,01+/-0,002$ & $0,01+/-0,002$ & $0,011+/-0,0016$ \\
DFG $<60 \mathrm{ml} / \mathrm{min} / 1,73 \mathrm{~m}^{2}$ & $0,01+/-0,002$ & $0,008+/-0,002$ & $0,009+/-0,002$ \\
DFG $\left[60 ; 90 \mathrm{ml} / \mathrm{min} / 1,73 \mathrm{~m}^{2}[\right.$ & $0,011+/-0,001$ & $0,011+/-0,0012$ & $0,011+/-0,0011$ \\
DFG $\geq 90 \mathrm{ml} / \mathrm{min} / 1,73 \mathrm{~m}^{2}$ & $0,01+/-0,002$ & $0,014+/-0,002$ & $0,014+/-0,0019$ \\
\hline
\end{tabular}


Tableau 14 : Comparaison des coefficients de corrélation entre $1 /$ créatininémie $(1 / \mu \mathrm{mol})$ et $\mathrm{DFG}$ $\left(\mathrm{ml} / \mathrm{min} / 1,73 \mathrm{~m}^{2}\right)$ obtenu avec MDRD et CKD-EPI chez les diabétiques de type 2.

\begin{tabular}{lccc}
\hline & \multicolumn{3}{c}{ DT2 $(\mathbf{n}=\mathbf{7 0})$} \\
\hline DFG $\left[0 ; 160 \mathrm{ml} / \mathrm{min} / 1,73 \mathrm{~m}^{2}[\right.$ & 0,851 & $\mathbf{r}(\mathbf{C K D}-\mathbf{E P I} ; \mathbf{1 / C r})$ & $\mathbf{Z}_{\mathbf{0}} \boldsymbol{\alpha}>\mathbf{1 , 9 6}$ \\
DFG<90 $\mathrm{ml} / \mathrm{min} / 1,73 \mathrm{~m}^{2}$ & 0,817 & 0,873 & 0,49 \\
DFG $<60 \mathrm{ml} / \mathrm{min} / 1,73 \mathrm{~m}^{2}$ & 0,982 & 0,787 & 0,49 \\
DFG $\left[60 ; 90 \mathrm{ml} / \mathrm{min} / 1,73 \mathrm{~m}^{2}[\right.$ & 0,615 & 0,961 & 2,26 \\
DFG $\geq 90 \mathrm{ml} / \mathrm{min} / 1,73 \mathrm{~m}^{2}$ & 0,602 & 0,526 & 0,77 \\
\hline
\end{tabular}

Tableau 15: Comparaison des coefficients de corrélation entre $1 /$ créatininémie $(1 / \mu \mathrm{mol})$ et $\mathrm{DFG}$ $\left(\mathrm{ml} / \mathrm{min} / 1,73 \mathrm{~m}^{2}\right)$ obtenu avec CG et CKD-EPI chez les diabétiques de type 2 .

\begin{tabular}{lccc}
\hline \multicolumn{4}{c}{ DT2 $(\mathbf{n}=\mathbf{7 0})$} \\
\hline & CG & CKD-EPI & $\mathrm{T}_{0} \alpha>1,96$ \\
\hline $\mathrm{DFG} \geq 90 \mathrm{ml} / \mathrm{min} / 1,73 \mathrm{~m}^{2}$ & $21,43 \%$ & $20 \%$ & 0,21 \\
$\mathrm{DFG}<90 \mathrm{ml} / \mathrm{min} / 1,73 \mathrm{~m}^{2}$ & $78,57 \%$ & $80 \%$ & 0,21 \\
$\mathrm{DFG}\left[60 ; 90 \mathrm{ml} / \mathrm{min} / 1,73 \mathrm{~m}^{2}[\right.$ & $42,86 \%$ & $65,71 \%$ & 2,71 \\
$\mathrm{DFG}<60 \mathrm{ml} / \mathrm{min} / 1,73 \mathrm{~m}^{2}$ & $35,71 \%$ & $14,29 \%$ & 2,91 \\
\hline
\end{tabular}

Tableau 16: Comparaison des coefficients de corrélation entre $1 /$ créatininémie $(1 / \mu \mathrm{mol})$ et $\mathrm{DFG}$ $\left(\mathrm{ml} / \mathrm{min} / 1,73 \mathrm{~m}^{2}\right)$ obtenu avec CG et CKD-EPI chez les diabétiques de type 2 .

\begin{tabular}{lccc}
\hline & \multicolumn{3}{c}{ DT2 $(\mathbf{n}=\mathbf{7 0})$} \\
\hline & $\mathbf{r}(\mathbf{C G} ; \mathbf{1} / \mathbf{C r})$ & $\mathbf{r}(\mathbf{C K D}-\mathbf{E P I} ; \mathbf{1} / \mathbf{C r})$ & $\mathbf{Z}_{\mathbf{0}} \boldsymbol{\alpha}>\mathbf{1 , 9 6}$ \\
\hline DFG $\left[0 ; 160 \mathrm{ml} / \mathrm{min} / 1,73 \mathrm{~m}^{2}[\right.$ & 0,689 & 0,873 & 2,88 \\
DFG $<90 \mathrm{ml} / \mathrm{min} / 1,73 \mathrm{~m}^{2}$ & 0,545 & 0,787 & 2,61 \\
DFG $<60 \mathrm{ml} / \mathrm{min} / 1,73 \mathrm{~m}^{2}$ & 0,632 & 0,961 & 7,02 \\
DFG $\left[60 ; 90 \mathrm{ml} / \mathrm{min} / 1,73 \mathrm{~m}^{2}[\right.$ & 0,157 & 0,526 & 2,46 \\
DFG $\geq 90 \mathrm{ml} / \mathrm{min} / 1,73 \mathrm{~m}^{2}$ & 0,729 & 0,808 & 1,13 \\
\hline
\end{tabular}

\section{DISCUSSION}

Le DFG est considéré comme étant le meilleur marqueur de la fonction rénale (Tomáš et al., 2014). Les formules de CG, du MDRD simplifiée et du CKD-EPI ont été élaborées pour estimer le DFG (Cockroft et Gault, 1976 ; Levey et al., 2006 ; 2009).

La formule de CG a été établie à partir 249 sujets (Cockroft et Gault, 1976). Elle intègre des données anthropométriques (âge, sexe, poids) et la créatinine sérique. Elle a été validée à partir d'une méthode de «référence » qui est la Clcr. Or, la Clcr nécessite une collecte des urines de 24 heures qui peut initier des erreurs grossières en raison de mécanismes subjectifs ou objectifs (Earley et al., 2012 ; Xioguang et al., 2014 ).

La formule du MDRD a été développée à partir de 1304 caucasiens et 324 AfroAméricains atteints d'insuffisance rénale chronique (IRC) (Levey et al., 2006). La formule du MDRD pourrait ne pas être valable chez les sujets de race noire qui constituent une partie relativement faible de la 
population de cette étude (Xioguang et al., 2014). La formule du MDRD simplifiée intègre des données anthropométriques (âge, sexe, race) et la créatinine sérique et elle est plus précise que la formule de CG pour les valeurs du DFG inférieures à $60 \mathrm{ml} / \mathrm{min} / 1,73$ $\mathrm{m}^{2}$ (Levey, 2006). Elle sous-estime les valeurs du DFG qui sont supérieures à $60 \mathrm{ml} / \mathrm{min} / 1,73$ $\mathrm{m}^{2}$ (Rigalleau et al., 2011).

La formule du CKD-EPI a été élaborée à partir de 8254 sujets. Elle a été validée à partir de 3896 sujets. Ces sujets étaient atteints ou non d'IRC. La formule du CKDEPI a la précision de la formule du MDRD simplifiée pour les valeurs du DFG inférieures à $60 \mathrm{ml} / \mathrm{min} / 1,73 \mathrm{~m}^{2}$. Elle prend en compte les mêmes paramètres que le MDRD mais elle est moins biaisée que la formule du MDRD simplifiée pour les valeurs du DFG supérieures à $60 \mathrm{ml} / \mathrm{min} / 1,73 \mathrm{~m}^{2}$ (Levey et al., 2009).

Les trois formules prennent en compte des données anthropométriques avec des incertitudes particulières chez les sujets diabétiques du fait du large éventail de DFG, d'IMC et de l'effet propre de la glycémie (Rigalleau et al., 2011).

Chez les DT1, l'étude a montré que les formules du CKD-EPI et du MDRD simplifiée conduisent à des moyennes superposables de DFG $(91,11 \pm 25,91$ versus $89,96 \pm 26,21 \mathrm{ml} / \mathrm{min} / 1,73 \mathrm{~m}^{2} ; \mathrm{p}=0,81$ ) (Tableau 5).

Chez les DT2, les deux formules (CKD-EPI, MDRD) révèlent également les mêmes moyennes de DFG $(76,23 \pm 19,06$ versus $78,61 \pm 19,04 \mathrm{ml} / \mathrm{min} / 1,73 \mathrm{~m}^{2} ; \mathrm{p}=0,46$ ) (Tableau 11).

Ces résultats confirment ceux de Fontela et al. (2014) qui ont montré que les formules du CKD-EPI et du MDRD simplifiées donnaient les mêmes moyennes de DFG chez 94 femmes DT2 (66.5 \pm 20.4 versus $66.2 \pm 22.1$; NS) et chez 54 hommes DT2 (74.5 \pm 20.5 versus $79.3 \pm 26.4 ; \mathrm{NS}$ ).

Chez les DT1, les formules de CKDEPI et du MDRD simplifiées conduisent exactement à la même proportion de sujets à
DFG réduit $\left(47,46 \%\right.$ versus $47,46 \% ; \mathrm{T}_{0} \alpha=$ 0) (Tableau 6).

Le constat ne change pas chez les DT2 où les formules de CKD-EPI et du MDRD révèlent aussi la même proportion de sujets à DFG réduit $\left(80 \%\right.$ versus $\left.80 \% ; \mathrm{T}_{0} \alpha=0\right)$. (Tableau 12).

Ces résultats corroborent ceux de Fontela et al. (2014) révélant, chez 146 DT2, que les proportions de sujets à $\mathrm{DFG}$ réduit obtenues avec les formules du CKD-EPI et du MDRD simplifiée sont superposables $(34,2 \%$ vs $36,3 \%)$. Ils n'ont pas précisé le p.

Les formules du CKD-EPI et du MDRD conduisent aussi à des coefficients de corrélation entre le DFG et le $1 / \mathrm{Cr}$ superposables chez les DT1 $(0,865$ versus 0,$858 ; Z_{0} \alpha=0,14$ ) (Tableaux 5, 6, 7, 8).

De même, chez les DT2, elles donnent les mêmes coefficients de corrélation entre le DFG et le $1 / \mathrm{Cr}\left(0,787\right.$ versus 0,$817 ; \mathrm{Z}_{0} \alpha=$ 0,49) (Tableaux $13 ; 14$ ).

Ces résultats sont en accord avec ceux de Fontela (2014) qui chez 146 DT2, trouvent des coefficients de corrélation similaires entre la $\mathrm{Cr}$ et le DFG pour CKD-EPI et MDRD (($0,89$ vs $-0,87)$.

Il semble donc, à la lumière des arguments qui viennent d'être évoqués, que le DFG peut être déterminé chez les sujets diabétiques de type 1 comme chez les sujets diabétiques de type 2 en utilisant indifféremment la formule du MDRD simplifiée ou celle du CKD-EPI.

L'équivalence de ces deux formules chez les DT1 et chez les DT2 s'expliquerait par le fait qu'elles s'appuient toutes les deux sur les mêmes variables que sont la créatininémie, l'âge, le sexe et la race (Levey et al., 2009).

Comme les formules du CKD-EPI et du MDRD simplifiée sont équivalentes chez les DT1 et chez les DT2, c'est la formule du CKD-EPI qui a été comparée à celle de CG (Tableaux $5 ; 7 ; 9 ; 10 ; 11 ; 13 ; 15 ; 16$ ).

Chez les DT1, la formule de CG permet d'obtenir une valeur moyenne du DFG superposable à celle déterminée à l'aide de la 
formule du CKD-EPI $\quad(82,61 \pm 26,57$ $\mathrm{ml} / \mathrm{min} / 1,73 \quad \mathrm{~m}^{2} \quad$ versus $91,11 \pm 25,91$ $\mathrm{ml} / \mathrm{min} / 1,73 \mathrm{~m}^{2} ; \mathrm{p}=0,81$ ) (Tableau 5).

Chez les DT2, la formule de CG donne une valeur moyenne du DFG statistiquement superposable à celle obtenue avec la formule du CKD-EPI (71,87+/-24,23 versus 76,23+/$\left.19,06 \mathrm{ml} / \mathrm{min} / 1,73 \mathrm{~m}^{2} ; \mathrm{p}=0,24\right)$ (Tableau 11).

Ces résultats confirment ceux obtenus par Rigalleau et al. (2005) chez 50 DT1. En effet, pour eux, la formule de $\mathrm{CG}$ et la formule du MDRD simplifiée donnent des moyennes ne présentant aucune différence statistiquement significative $(66,6+/-35,4$ $\mathrm{ml} / \mathrm{min} / 1,73 \quad \mathrm{~m}^{2}$ versus $62,4 \quad+/-$ $29,7 \mathrm{ml} / \mathrm{min} / 1,73 \mathrm{~m}^{2}$; NS).

Par contre, Vervoort et al. (2002) ont trouvé que la formule de $\mathrm{CG}$ est plus précise que la formule du MDRD chez les DT1. En effet, leurs résultats montrent que la différence de médiane entre la clairance de l'inuline et le DFG calculé était plus faible en utilisant la formule de CG plutôt que celle du MDRD simplifiée $\left(11,8 \mathrm{ml} / \mathrm{min} / 1,73 \mathrm{~m}^{2}\right.$ versus 18,8 $\mathrm{ml} / \mathrm{min} / 1,73 \mathrm{~m}^{2}$ ).

Chez les DT2, les résultats obtenus dans le présent travail ne confirment pas ceux de Rigalleau et al. En effet, ces auteurs ont trouvé que la formule de CG donne, chez 110 DT2, une moyenne de DFG significativement supérieure à celle obtenue avec la formule du MDRD simplifiée $(65,2+/-38,5 \mathrm{ml} / \mathrm{min} / 1,73$ versus $51,2+/-22,0 \mathrm{ml} / \mathrm{min} / 1,73 ; \mathrm{p}<0,001)$.

Par ailleurs, il est apparu, chez les DT1, que la proportion de sujets à $\mathrm{DFG}$ réduit $(<90$ $\mathrm{ml} / \mathrm{min} / 1,73 \mathrm{~m}^{2}$ ) est statistiquement plus importante avec la formule de Cockroft et Gault plutôt qu'avec celle du CKD-EPI $\left(66,10 \%\right.$ versus $\left.47,46 \% ; \quad \mathrm{T}_{0} \alpha=2,05\right)$ (Tableau 9).

Par contre, chez les DT2, la proportion de sujets à DFG réduit obtenue avec la formule du CKD-EPI est superposable à celle obtenue avec la formule de CG (80\% versus 78,57\%; $\mathrm{T}_{0} \alpha=0,21$ ) (Tableau 15).

Ces résultats ne confirment pas ceux de Fontela et al. (2014) qui ont rapporté une proportion de sujets à DFG réduit plus faible avec la formule de CG (25\%) qu'avec la formule du CKD-EPI (34,2\%).

Chez les DT1, il est apparu que, lorsque les DFG calculés avec la formule du CKD-EPI sont inférieurs à $90 \mathrm{ml} / \mathrm{min} / 1,73 \mathrm{~m}^{2}$, la corrélation entre le $1 / \mathrm{Cr}$ et le DFG est de $\mathrm{r}=$ 0,865 . Cette corrélation ne varie pas lorsque les DFG sont calculés avec la formule de CG ( $r=0,793)$. En effet, la différence entre les deux coefficients de corrélation n'est pas statistiquement significative $(0,793$ versus 0,$865 ; Z_{0} \alpha=1,23$ ) (Tableau 10).

Par contre, chez les DT2, lorsque les DFG déterminés avec la formule du CKD-EPI sont inférieurs à $<90 \mathrm{ml} / \mathrm{min} / 1,73 \mathrm{~m}^{2}$, la corrélation entre le $1 / \mathrm{Cr}$ et le DFG est de $\mathrm{r}=$ 0,787. Cette corrélation devient faible lorsque les DFG sont déterminés avec la formule de $\mathrm{CG}(\mathrm{r}=0,545)$. La différence entre ces deux coefficients de corrélation est, en effet, statistiquement significative $(0,787$ versus 0,$545 ; Z_{0} \alpha=2,61$ ) (Tableau 16).

Si les DFG sont compris entre 90 et 60 $\mathrm{ml} / \mathrm{min} / 1,73 \mathrm{~m}^{2}$, la corrélation entre $1 / \mathrm{Cr}$ et DFG devient presque inexistant $(r=0,157)$ avec la formule de CG chez les DT2. Par contre, lorsque les DFG sont déterminés avec la formule du CKD-EPI chez les DT2, la corrélation entre le $1 / \mathrm{Cr}$ et le DFG demeure (r $=0,545)$. La différence entre ces deux coefficients de corrélation est statistiquement significative $\left(\mathrm{Z}_{0} \alpha=2,46\right)$ (Tableau 16$)$.

Ces résultats confirment ceux de Rigalleau et al. (2005) et de Fontela et al. (2014). En effet, selon Rigalleau et al. (2005), le coefficient de corrélation entre la clairance de l'EDTA- ${ }^{51} \mathrm{Cr}$ et le DFG était plus faible avec un DFG était calculé avec la formule de CG plutôt qu'avec la formule du MDRD simplifiée chez les DT1 $(0,72$ versus 0,83$)$ et chez les DT2 (0,76 versus 0,83), sans précision du p. Pour Fontela et al. (2014), le coefficient de corrélation entre la $\mathrm{Cr}$ et le DFG, obtenu chez des DT2, était plus petit lorsque le DFG est calculé avec la formule de CG plutôt qu'avec la formule du CKD-EPI (0,64 versus -0,83). Avec les arguments précédents, il semble qu'il devient possible de 
suggérer que, pour estimer le DFG chez les sujets diabétiques de type 2 afin de déceler une réduction du DFG, la formule du CKDEPI est plus appropriée que celle de CG (Rigalleau et al., 2005 ). Néanmoins, malgré toutes ses limites, la formule de CG aurait encore sa place dans la détermination du DFG du DT1, si l'objectif est de dépister toute diminution du DFG chez les DT1 (Drion I et al., 2011 ; Vervoort et al., 2002) (Tableau 9).

La formule de Cockroft et Gault a été plus appropriée pour dépister une diminution du DFG chez les sujets diabétiques de type 1 de notre étude que la formule du CKD-EPI et la formule du MDRD modifiée (Tableaux 5 ; $9,10)$. Ce constat est également celui de Drion et al. (2011) et de Vervoort et al. (2002). Cependant, certains auteurs ont émis des réserves à ce constat (Rigalleau et al., 2005 ; Drion et al., 2011). Toutefois, c'est un constat qui pourrait être expliqué. En effet, comme les formules du MDRD simplifiée et du CKD-EPI, la formule de CG intègre les variables créatininémie, âge, et sexe ; en plus, elle emploie la variable poids qui a sa place, dans la formule, au niveau du numérateur. Donc, quand les autres variables communes (créatininémie, âge, et sexe) sont fixées, la diminution du poids entraîne inéluctablement la réduction du DFG. Or, les sujets diabétiques de type 1 de l'étude ont un poids dont la valeur moyenne $(64,68 \pm 10,71 \mathrm{~kg}$ versus $\left.68,26 \pm 16,95 \mathrm{~kg}, \mathrm{p}=1,8 \cdot 10^{-1}\right)$, si elle n'est pas inférieure, reste statistiquement égale à celle obtenue chez les sujets témoins. Ils ont aussi un IMC qui est statistiquement superposable à celui des sujets témoins $\left(22,61 \pm 3,92 \mathrm{~kg} / \mathrm{m}^{2}\right.$ versus $23,39 \pm 4,93 \mathrm{~kg} / \mathrm{m}^{2}, \mathrm{p}$ $\left.=3,4 \cdot 10^{-1}\right)$. Les sujets diabétiques de type 1 et les sujets témoins, de même race, ont la même moyenne d'âge $(35,39 \pm 11,28$ versus $\left.35,6 \pm 12,91, p=9,2 \cdot 10^{-1}\right)$ et le même sex ratio $(\mathrm{H} / \mathrm{F})(0,39$ versus 0,$45 ; \mathrm{T} 0 \alpha=0,66)$.

En recherchant une réduction du DFG chez les DT2 de notre étude, la formule du CKD-EPI a été plus appropriée que celle de CG (Tableaux $11 ; 15 ; 16)$. Ce constat a été fait par plusieurs auteurs dont Rigalleau et al. (2005).

Ce constat s'expliquerait par le fait que dans les formules du MDRD modifiée, du CKD-EPI et de Cockroft et Gault, il est noté la présence des variables âge, sexe, race et créatininémie, mais c'est seulement dans la formule de Cockroft et Gault que la variable poids est retrouvée. C'est serait la variable poids qui rendrait la formule de Cockroft et Gault moins efficace pour estimer le DFG que la formule du CKD-EPI chez les sujets diabétiques de type 2 . Il est bien connu que la créatininémie augmente avec la masse musculaire puisque la créatinine est un catabolite de la créatine-phosphate, molécule de réserve énergétique pour la cellule musculaire; ce qui fait que plus les cellules musculaires sont nombreuses, plus la production de créatinine est importante. Autant l'accroissement du poids est dû à une augmentation seulement de la masse musculaire, autant cela se traduit par une augmentation de la créatininémie. Etant donné que le poids est au numérateur et la créatininémie au dénominateur dans la formule de Cockroft et Gault, l'augmentation simultanée et proportionnelle des deux variables poids et créatininémie laisse constante la valeur du DFG. Cependant, le poids d'un sujet n'est pas seulement dû à la masse musculaire, il est également tributaire de la masse osseuse et de la masse adipeuse (Batsis et al., 2014). Dans notre étude, il a été noté une augmentation du poids $(71,8 \pm 13,23$ $\mathrm{kg}$ versus $\left.64,68 \pm 10,71 \mathrm{~kg} ; \mathrm{p}=1,2.10^{-3}\right)$, de l'IMC $\left(25,77 \pm 4,84 \mathrm{~kg} / \mathrm{m}^{2}\right.$ versus $22,61 \pm 3,92$ $\left.\mathrm{kg} / \mathrm{m}^{2} ; \mathrm{p}=9,8.10^{-5}\right)$ et de l'IMG $(36,90 \pm 7,27$ $\%$ versus $25,66 \pm 9,35 \% ; \mathrm{p}=3,87.10^{-12}$ ) chez les sujets diabétiques de type 2 par rapport aux sujets diabétiques de type 1 . Cependant, les valeurs moyennes du poids et de l'IMC ne dépassent pas les limites supérieures de référence qui sont à $70 \mathrm{~kg}$ pour le poids et à $25 \mathrm{~kg} / \mathrm{m}^{2}$ pour l'IMC (Batsis et al., 2014). Par contre, la valeur moyenne de l'IMG est à $36,90 \%$ dépassant largement la limite supérieure de référence qui est à 30\% (Batsis 
et al., 2014). L'augmentation du poids chez les sujets diabétiques de type 2 de l'étude est par conséquent plus tributaire de la masse adipeuse que de la masse musculaire (Tableau $2)$. Or, les cellules adipeuses ne sont pas productrices de créatinine, l'élévation du poids s'accompagne donc d'une augmentation disproportionnée de la créatininémie. Ce qui fait que d'une part, il existe une faiblesse considérable dans la corrélation positive entre le $1 / \mathrm{Cr}$ et le DFG selon CG qui est à $\mathrm{r}=0,157$ contre $r=0,526$ avec la formule du CKD-EPI avec une différence significative $(\mathrm{ZO} \alpha=2,46)$ chez les sujets diabétiques de type 2 à DFG compris entre 90 et $60 \mathrm{ml} / \mathrm{min} / 1,73$ $\mathrm{m}^{2}$ (Tableau 16); d'autre part, l'augmentation du numérateur de la formule de Cockroft et Gault, à savoir le poids, qui n'est pas suivie par l'élévation proportionnelle du dénominateur (la créatininémie) entraîne une surestimation du DFG qui abaisse, par exemple, la proportion de sujets diabétiques de type 2 à DFG $<90 \mathrm{ml} / \mathrm{min} / 1,73 \mathrm{~m}^{2}(42,86 \%$ avec CG contre $65,71 \%$ avec CKD-EPI ; T0 $\alpha$ $=2,71)$ (Tableau 15).

\section{Conclusion}

$\mathrm{Au}$ terme de l'étude, qui avait pour objectif de rechercher la mieux adaptée parmi les formules de CG, du MDRD simplifiée ou du CKD-EPI pour estimer le DFG des DT1 et des DT2, il a été retenu que :

- chez les sujets diabétiques de type 1 , il est beaucoup plus indiqué de calculer le DFG avec la formule de CG plutôt qu'avec celle du CKD-EPI ou celle MDRD simplifiée. La formule de CG, malgré toutes ses limites, pourrait encore être utile pour estimer le DFG des DT1.

- chez les sujets diabétiques de type 2, la formule du CKD-EPI, équivalente à celle du MDRD modifiée, demeure plus appropriée que la formule de CG pour estimer le DFG.

Cependant, l'utilisation des formules d'estimation du DFG pouvant paraître rébarbative, ne serait-il pas plus pratique de recourir à d'autres paramètres tels la cystatinémie-C ?

\section{REFERENCES}

Batsis JA, Mackenzie TA, Barre LK, LopezJimenez F, Bartels SJ. 2014. Sarcopenia, sarcopenic obesity and mortality in older adults: results from the National Health and Nutrition Examination Survey III. European Journal of Clinical Nutrition, 68: 1001-1007.

Battisti WP, Palmisano J, Keane WE. 2003. Dyslipidemia in patients with type 2 diabetes. relationships between lipids, kidney disease and cardiovascular disease. ClinChem Lab Med., 41(9): 1174-1181.

Cockroft DW, Gault MH. 1976. Prediction of creatinine clearance from serum creatinine. Nephron., 16: 31-41.

Deurenberg P, Weststrate JA, Seidell JC. 1991. Body mass index as a measure of body fatness: age- and sex-specific prediction formulas. Br. J. Nutr., 65(2): 105-114.

Drion I, Joosten H, Santing L, Logtenberg SJ, Groenier KH, Lieverse AG, Kleefstra N, Bilo HJ. 2011. The Cockcroft-Gault: a better predictor of renal function in an overweight and obese diabetic population. Obes Facts., 4(5): 393-399.

Earley A, Miskulin D, Lamb EJ, Levey AS, Uhlig K. 2012. Estimating equations for glomerular filtration rate in the era of creatinine standardization: a systematic review. Ann Intern Med., 156(11): 785795.

Fontela PC, Winkelmann ER, Ott JN, Uggeri DP. 2014. Estimated glomerular filtration rate in patients with type 2 diabetes mellitus. Rev Assoc Med Bras., 60(6): 531-537.

Halimi JM. 2012. The emerging concept of chronic kidney disease without clinical proteinuria in diabetic patients. Diabetes Metab., 38(4): 291-297.

Hermida-Cadahia EF, Lampon N, Tutor JC. 2012. Impact of creatinine production on the agreement between glomerular filtration rate estimates using cystatin $\mathrm{C}$ derived, and 4- and 6-variable 
Modification of Diet in Renal Disease (MDRD), and Chronic Kidney Disease Epidemiology Collaboration (CKD-EPI) equations. Ups J Med Sci., 117(4): 402410.

Jerums G, Elif E, Sianna P, Richard JM. 2012. Early glomerular filtration rate loss as a marker of diabetic nephropathy. European Endocrinology, 8(1): 27-31.

Levey AS, Coresh J, Greene T, Stevens L A, Zhang Y L, Hendriksen S, Van Lente F. 2006. Using standardized serum creatinine values in the modification of diet in renal disease study equation for estimating glomerular filtration rate. Ann Intern Med., 145(4): 247-254.

Levey AS, Stevens LA, Schmid CH, Zhang YL, Castro AF 3rd, Feldman HI, Kusek JW, Eggers P, Van Lente F, Greene T, Coresh J, CKD-EPI (Chronic Kidney Disease Epidemiology Collaboration). 2009. A new equation to estimate glomerular filtration rate. Ann Intern Med, 150: 604-612.

Rigalleau V, Lasseur C, Perlemoine C, Barthe N, Raffaitin C, Liu C, Chauveau P, Baillet-Blanco L, Bauvieux MC, Combe C, Gin H.2005. Estimation of glomerular filtration rate in diabetic subjects: Cockcroft formula or modification of Diet in Renal Disease study equation? Diabetes Care, 28: 838-843.

Rigalleau V, Beauvieux M-C, Gonzalez C, Raffaitin C, Lasseur C, Combe C, Chauveau P, De la Faille R, Rigothier C,
Barthe N, Gin H. 2011. Estimation of renal fonction in patients with diabetes. Diabetes \& Metabolism, 37: 359-366.

Stevens LA, Coresh J, Greene T, Levey AS. 2006. Assessing kidney function-measured and estimated glomerular filtration rate. $N$ Engl J Med, 354(23): 2473-2483.

Tomáš Ž, Branislav K, Ida P, Tomáš P, Anton K. 2014. Use of selected prediction equations (CG, MDRD4, CKD-EPI) in improving glomerular filtration rate assessment clinical practice in Slovakia. Cent Eur J Public Health, 22(1): 34-41.

Vervoort G, Willems HL, Wetzels JF. 2002. Assessment of glomerular filtration rate in healthy subjects and normoalbuminuric diabetic patients: validity of a new (MDRD) prediction equation. Nephrol Dial Transplant,17: 1909-13.

Xiaoguang Z, Gang Y, Niansong W, Qing X, Xiaohua S, Yan. 2014. Comparison of Seven Kinds of Calculation Methods on Glomerular Filtration Rate in Patients with Chronic Kidney Disease. Journal of Integrative Nephrology and Andrology, 1: 20-23.

Yasmin A, Hasniza ZH. 2015. Chronic Kidney Disease Screening Methods and Its Implication for Malaysia: An in Depth Review. Global Journal of Health Science, 7(4): 96-109. 